\title{
Supporting Information \\ The Role of the Dopants on the Morphological and Transport Properties of Spiro-MeOTAD Hole Transport Layer
}

Emilio J. Juarez-Perez, Matthew Leyden, Shenghao Wang, Luis K. Ono, Zafer Hawash, Yabing Qi*

Energy Materials and Surface Sciences Unit (EMSS), Okinawa Institute of Science and

Technology Graduate University (OIST), 1919-1 Tancha, Kunigami-gun, Onna-son, Okinawa 904-0495, Japan.

*Yabing.Qi@OIST.jp

\section{Mathematical proof and approximations used to relate phase during nap mode with material composite permittivity}

Phase variation asserted by electrostatic force gradients could be approximated to ${ }^{11}$

$$
\Delta \delta \cong \frac{Q}{2 k}\left(\frac{d^{2} C}{d z^{2}}\right) V^{2}
$$

where $V$ accounts mainly for the voltage difference applied, $Q$ and $k$ are the cantilever quality factor and spring constant, respectively. $C$ and $z$ are capacity and height, respectively. If there is no significant influence by work function and charging effects between tip and material in the four sample measurements. Considering that a plate capacitor between tip and sample is formed, the second derivative is

$$
\frac{d^{2} C}{d z^{2}}=2 \varepsilon_{r} \varepsilon_{0} \frac{a}{z^{3}}
$$

because all tests were performed using the same voltage, tip and delta height, the only variable tracked by phase would be the relative permittivity of the underlying material. By substituting (2) in equation no. (1) and knowing that $z$ and $V$ are constant through all experiments, the phase variation can be ascribed to the permittivity variation as

$$
\Delta \delta \cong \frac{Q \varepsilon_{r} \varepsilon_{0}}{k} \frac{a}{z^{3}} V^{2} \cong K \varepsilon_{r} \cong f\left(\varepsilon_{r}\right)
$$

Therefore the phase EFM maps qualitatively track changes in the material composite relative permittivity.

\footnotetext{
${ }^{1}$ Melin, T.; Zdrojek, M.; Brunel, D.: Electrostatic force microscopy and Kelvin force microscopy as a probe of the electrostatic and electronic properties of carbon nanotubes. In Scanning Probe Microscopy in Nanoscience and Nanotechnology; Springer, 2010; pp 89-128.
} 


\section{XRD for spiro-0 thin-film sample}

The observed diffraction peaks all belong to the diffractometer stage and there are no peaks associated with the spiro-MeOTAD film, see Figure S7. A large hump centered at $\sim 22^{\circ}$ in $2 \theta$ indicates that the spiro-MeOTAD film is mainly amorphous. Therefore, we conclude that the the spiro-MeOTAD film in our study has no long range ordering higher than 10-20 unit cells.

\section{Four-point probe conductivity measurements on high temperature annealed thin-film samples.}

Our standard procedure for thin film preparation includes a 24 hours drying step at room temperature and ambient conditions prior to any measurement. After this drying step, excessive weakly bound and/or physically-adsorbed chloroform molecules are expected to be removed. However, chloroform molecules that are co-crystallized within the spiro-MeOTAD structure are retained even after the drying step. According to our TG-DTA study, these solvated/crystallized $\mathrm{CH}_{3} \mathrm{Cl}$ molecules leave the thin film at around $140-160{ }^{\circ} \mathrm{C}$. Four point probe conductivity measurements were carried out on a new batch of spiro-MeOTAD thin-films before and after a heating treatment from room temperature to $160{ }^{\circ} \mathrm{C}\left(20{ }^{\circ} \mathrm{C} / \mathrm{min}\right.$ heating rate $)$ inside a $\mathrm{N}_{2}$ glovebox to mimic the same TG-DTA heating conditions. The results have been included in the support information, Table S2. Taking into account the experimental uncertainty in the sheet resistance measurements, no substantial changes in the sheet resistance were observed before and after the heating treatment. Therefore, the presence of solvated/crystallized chloroform molecules has only a minor effect on the conductivity of spiro-MeOTAD films.

\section{Theoretical comparison (structure and calculated FTIR) of two experimental spiro- MeOTAD conformers.}

The starting point structure for the theoretical calculations in this work was initially chosen from the spiro-MeOTAD crystal structure reported by Ganesan et al. [27] Figure S8 compares visually the calculated ground state spiro-MeOTAD structures using as starting point the spiro-MeOTAD structure from Ganesan et al. [27] and from the recently reported Shi et al. [28] structure, respectively. RMS data for non-hydrogen atoms is $0.516 \AA$ and $0.821 \AA$, respectively.

Analyzing both crystal structures for Spiro-MeOTAD, it can observed qualitatively several differences. The most important is that the Shi et al structure has not solvation molecule as reported by Ganesan et al. Another important difference is that both spiro-MeOTAD structure molecules extracted from the crystallographic data are not superimposable. Meanwhile the Spiro-MeOTAD molecule from Ganesan et al has a S4 improper axis (rotation-reflection) through the spiro carbon leaving the MeO-Ph-N branches connected to the fluorene in anti disposition. The structure from Shi et al has not such symmetry element and the MeO-Ph-N branches pairs of one of the fluorene units are in eclipsed disposition. The Shi et al Spiro-MeOTAD conformer has much less symmetry and it also can be observed by the bend of one of the fluorene units in the Shi et al structure. (See Figure S8). Interestingly, such bend could be due to 1) feble hydrogen bond ( $>\mathrm{O} \cdots \mathrm{H}-\mathrm{C}_{\mathrm{Ph}}, \mathrm{d}=$ 
$2.881 \AA$, highlighted with a red arrow in Figure S8) and 2) dihydrogen bond $\left(\mathrm{C}_{\mathrm{Me}}-\mathrm{H} \cdots \mathrm{H}-\mathrm{C}_{\mathrm{Ph}}, \mathrm{d}\right.$ $=2.404 \AA$, highlighted with a blue arrow in Figure S8) interactions between the branches. These intramolecular interactions cannot be observed for the Ganesan et al structure, neither the intermolecular $\pi-\pi$ stacking observed by Shi et al between neighbor fluorene units. RMS distance between non hydrogen atoms is significantly lower for the Ganesan et al structure than the Shi et al structure. It means that in this case the gas phase structure calculated is closer to experimental solid state than the calculated structured for the Shi et al experimental structure. Surprisingly, the DFT calculations reveal that the calculated structure from the Shi et al structure is $1.5 \mathrm{kcal} / \mathrm{mol}$ lower in energy than the Ganesan et al. but it is due to $2.7 \mathrm{kcal} / \mathrm{mol}$ of extra energy from the D3 dispersion correction for the Shi et al structure. Probably, it is due to the stabilizing behavior of the intramolecular hydrogen interactions in the Shi et al conformer.

About if there is major difference in the calculated IR spectra for both structures. Figure S9 show the calculated spectra for both theoretical structures. It can be observed that the major intensity difference is around $2 \%$ and depending of the vibration, the mismatch can be displaced to high frequency or low frequency, or even differences between sharpness or broadness of the vibration peak. In any case, these differences do not affect to the FTIR vibration chosen for the FTIR microscopy mappings. 

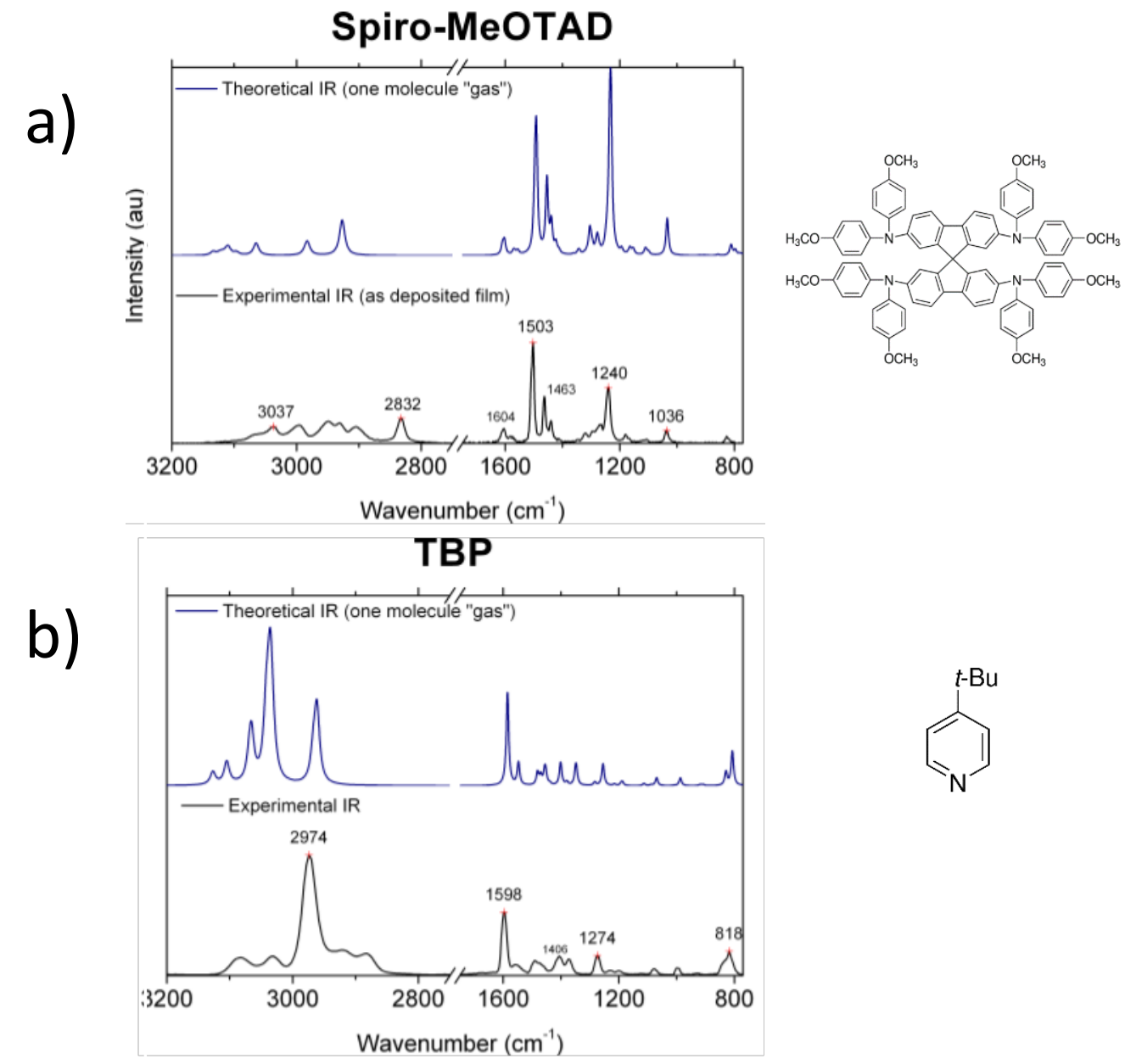<smiles>CC(C)(C)c1ccncc1</smiles>

c)

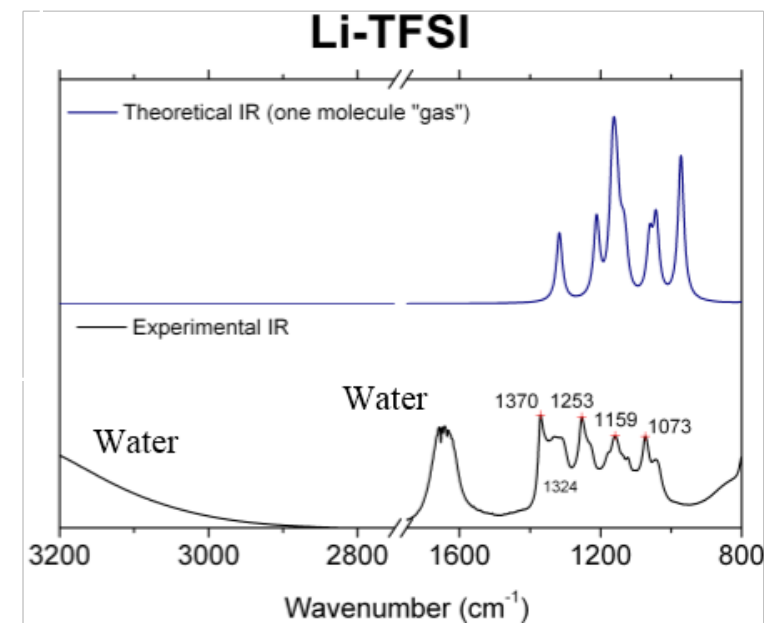

Figure S1. Simulated and experimental FTIR spectra for a) Spiro-MeOTAD, b) TBP and c) LiTFSI. Adjacent to the right, molecular structures of the compounds. 


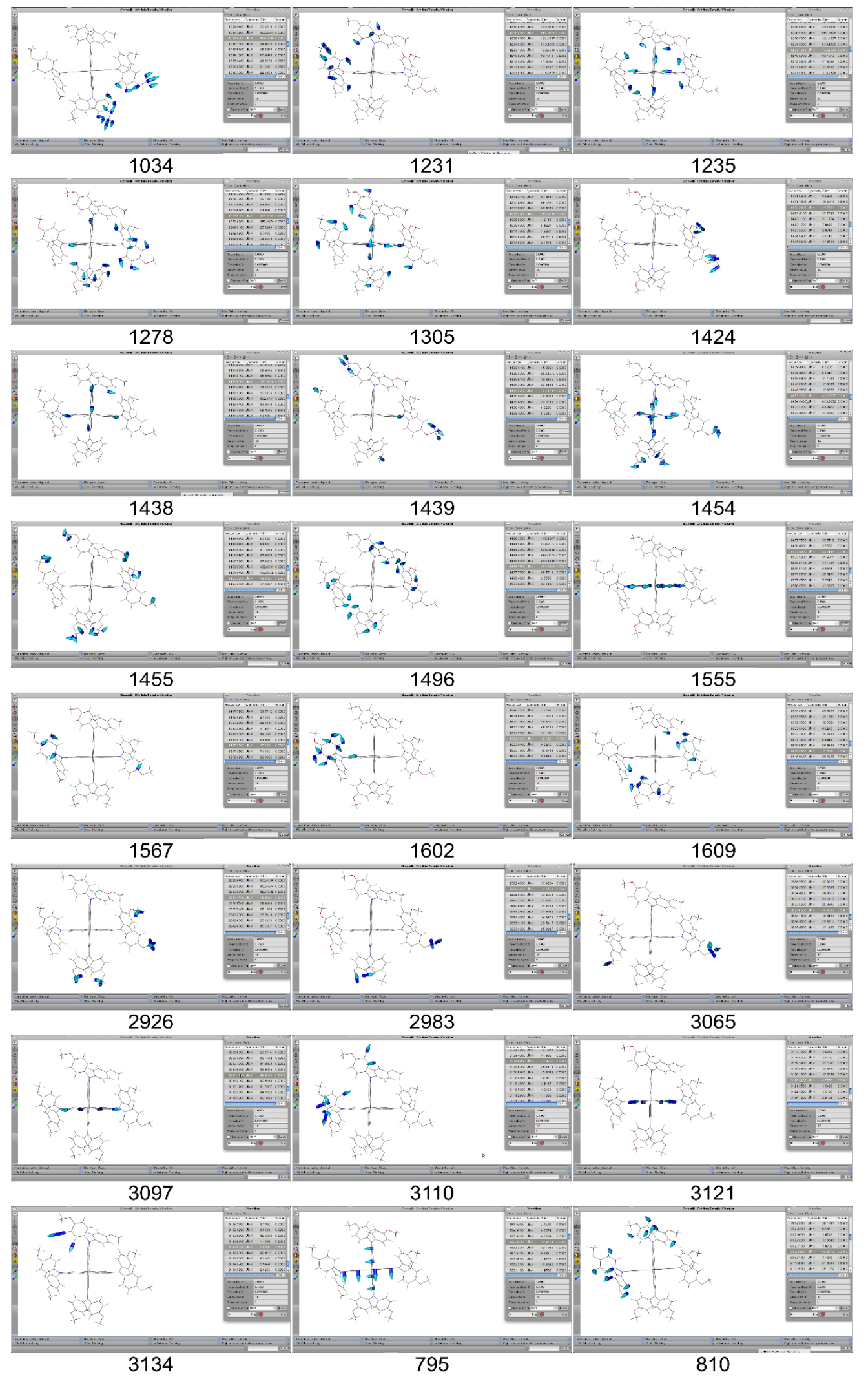

Figure

S2.

Selected vibrations for the calculated spiro-MeOTAD molecule. Numbers under the figures are wavenumbers of the corresponding vibrations. 


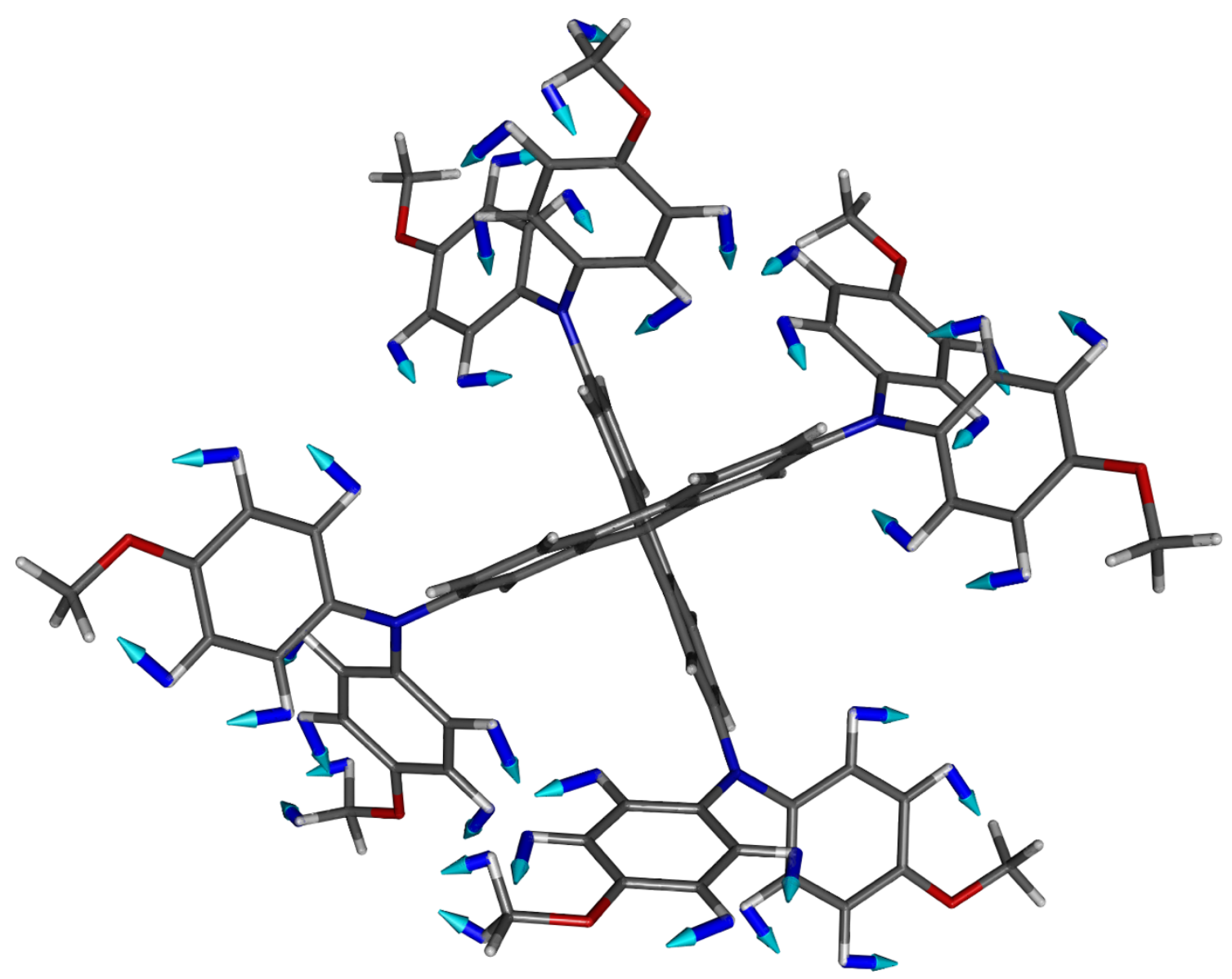

Figure S3. C-H rocking vibration at $1496 \mathrm{~cm}^{-1}$ in the calculated spiro-MeOTAD molecule. 


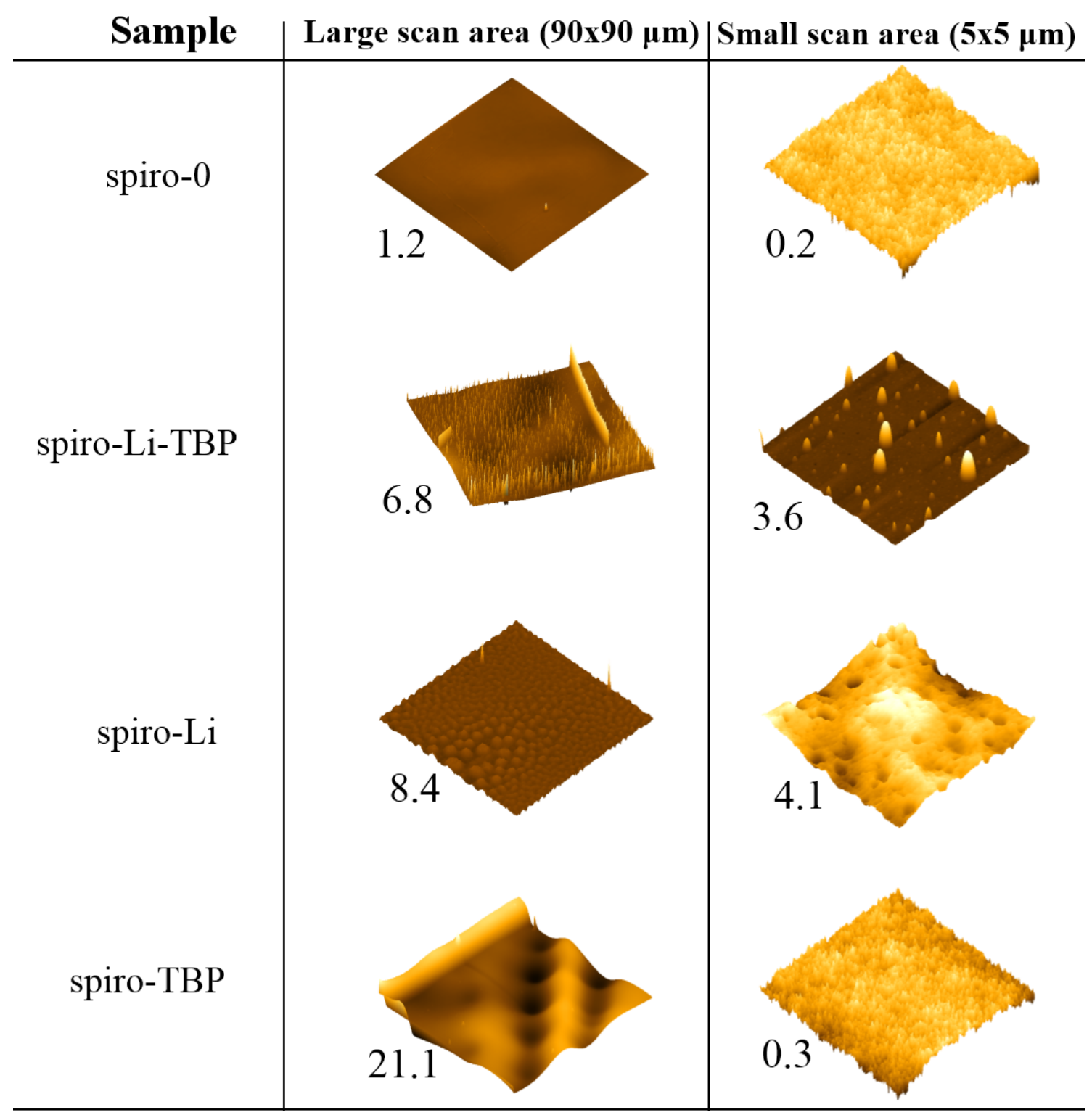

Figure S4. AFM (tapping mode) for the four thin films samples at two magnifications. Roughness is indicated for each measurement (nm). 

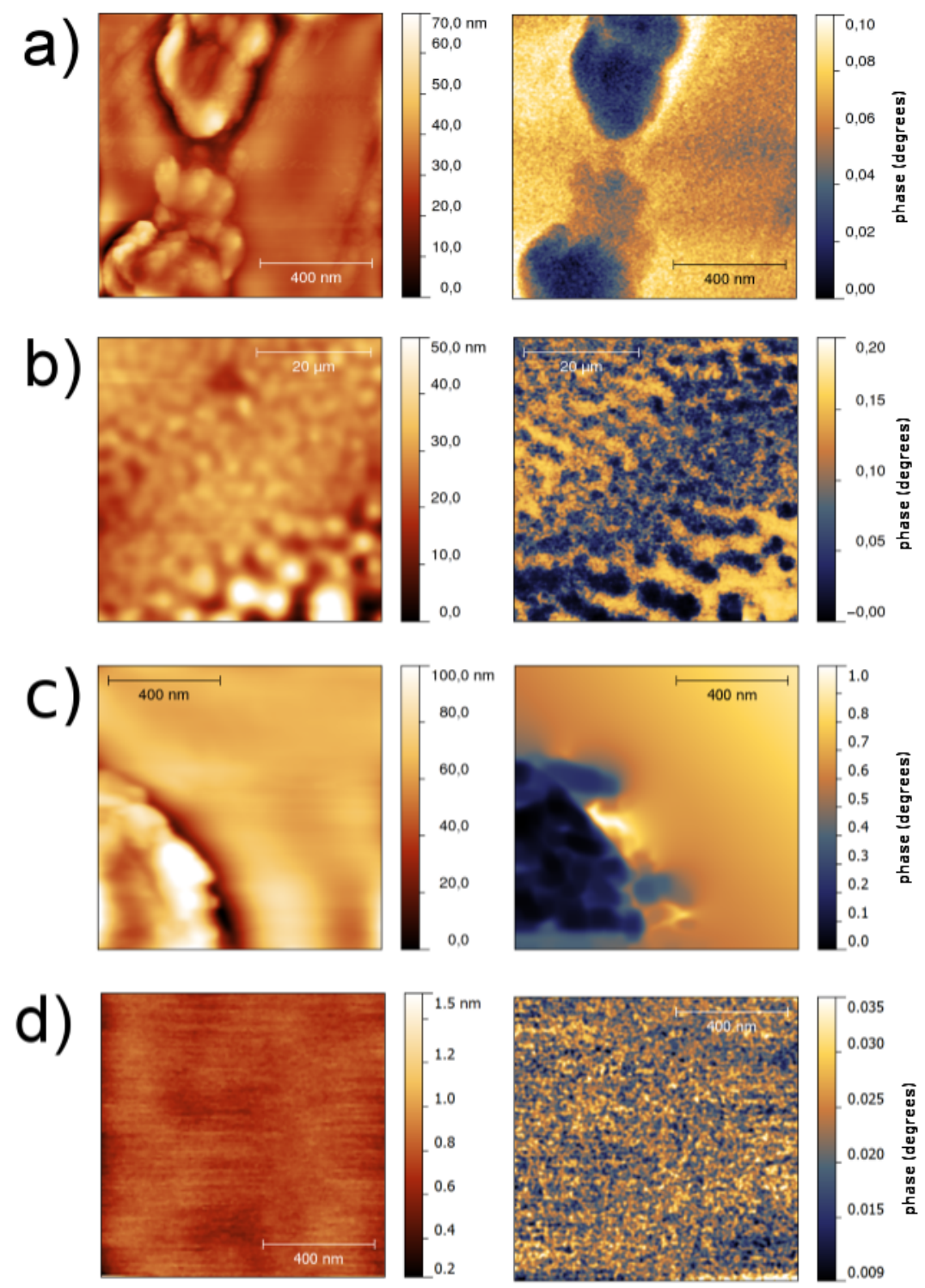

Figure S5. EFM (tapping mode left and nap mode right) for thin film samples at different magnifications. a) spiro-Li-TBP, b) low magnification spiro-Li, c) high magnification spiro-Li and d) high magnification spiro-TBP. 


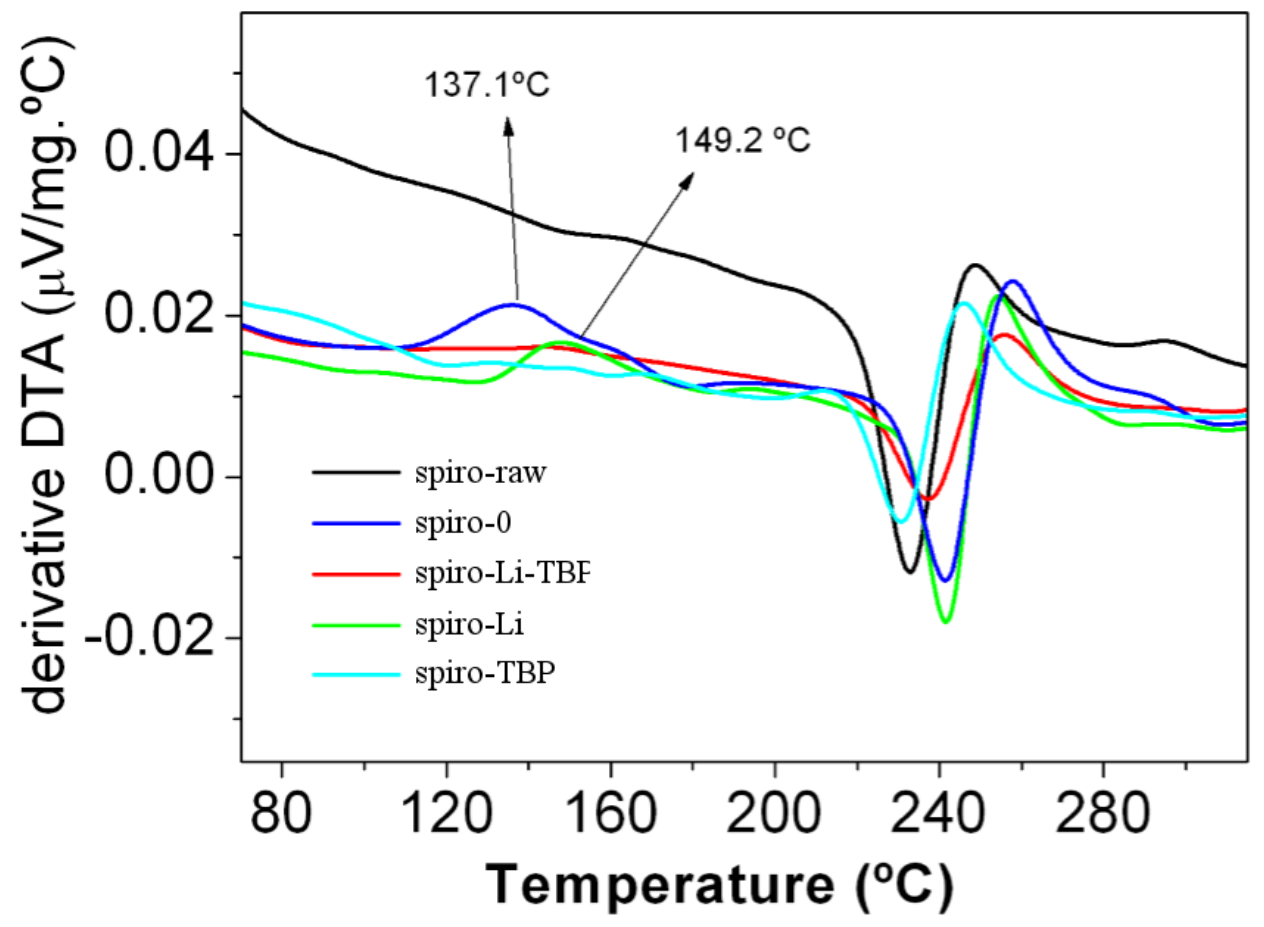

Figure S6. Derivative of the DTA curve for five bulk samples. Only spiro-Li and spiro0 show peaks (arrows) attributable to evaporation of chloroform (solvent) molecules. 


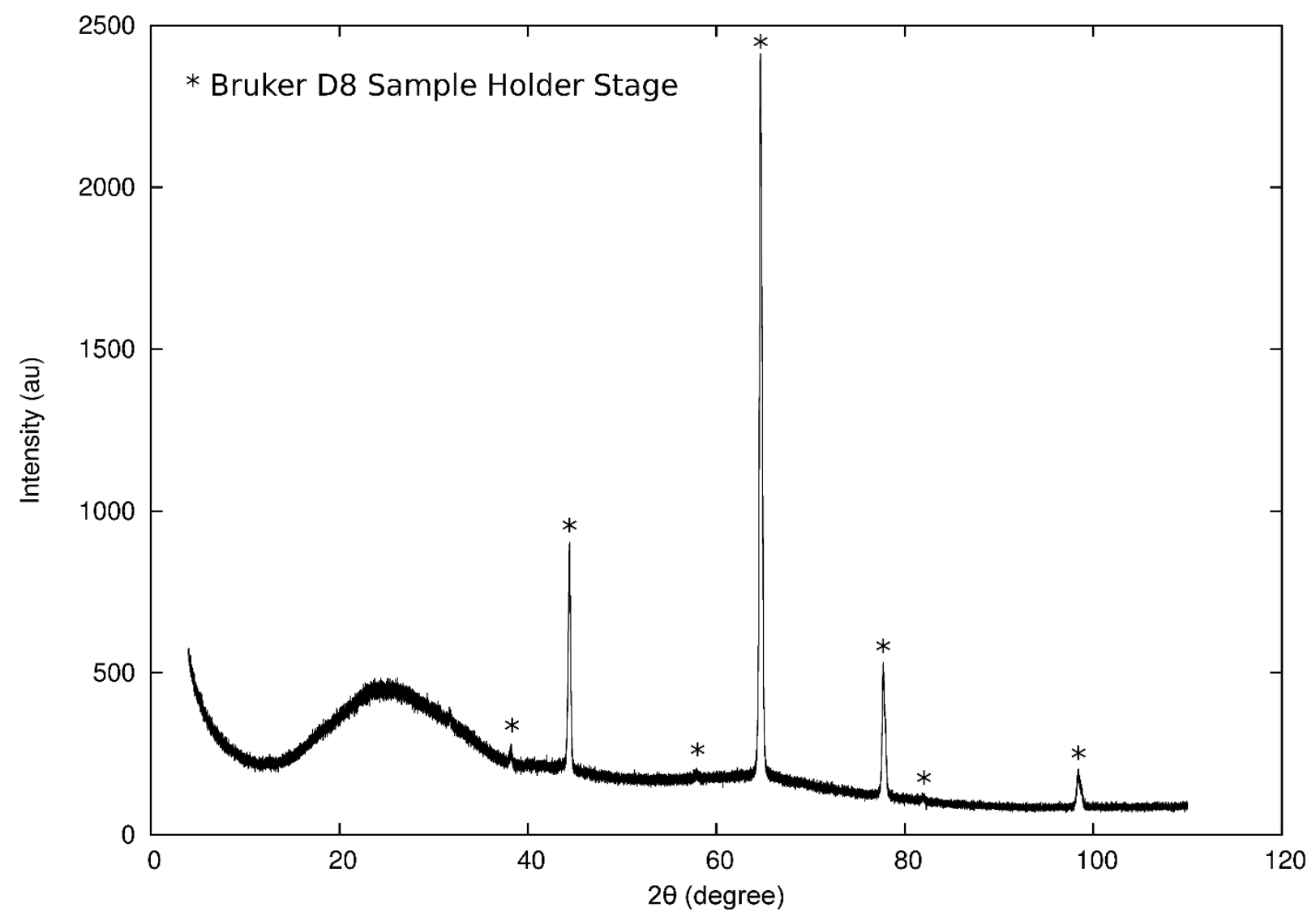

Figure S7. XRD pattern for thin-film sample spiro-0. Only the diffraction peaks from the sample holder stage were identified. 


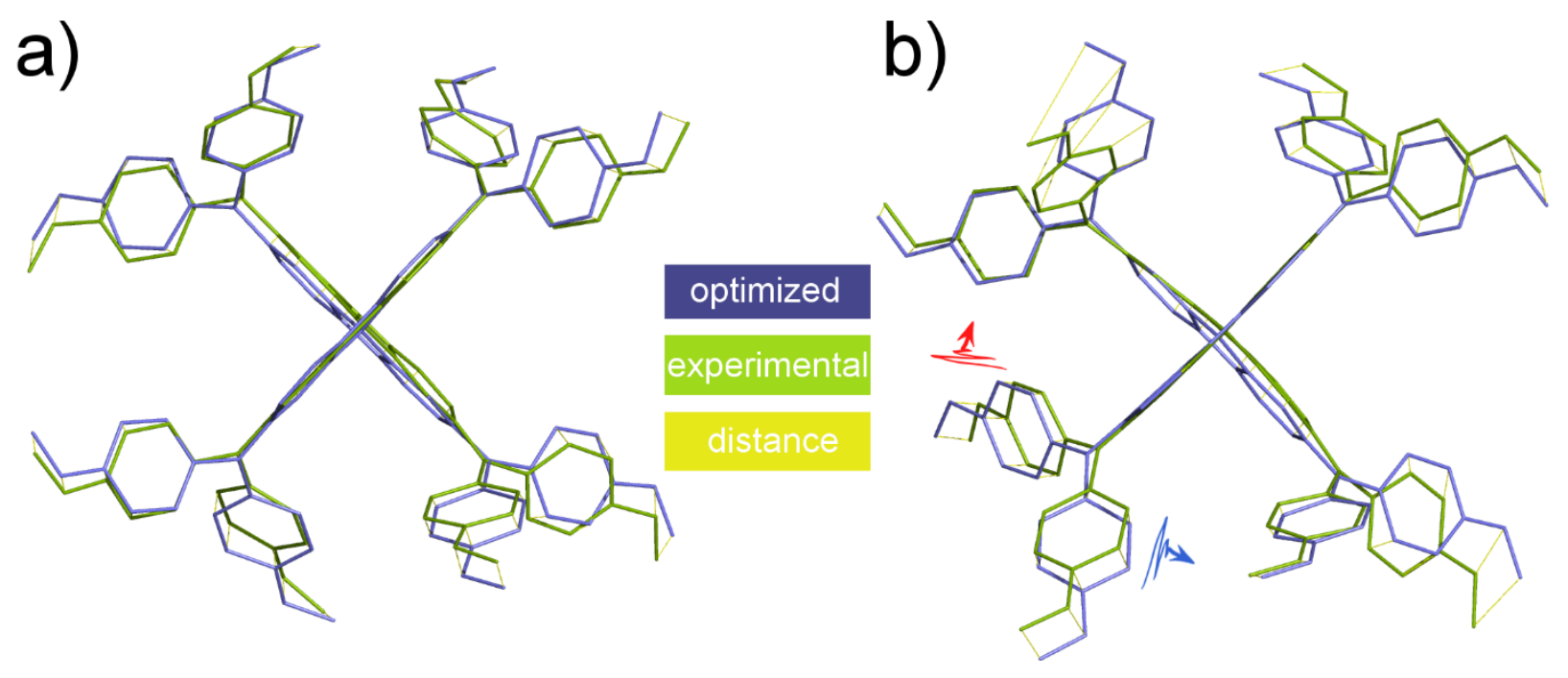

Figure S8. a) Aligned experimental (Ganesan et al [27]) and theoretical structure, RMS (non hydrogen atoms) $=0.516 \AA$, b) aligned experimental (Shi et al [28]) and theoretical structure, RMS (non hydrogen atoms) $=0.821 \AA$. 


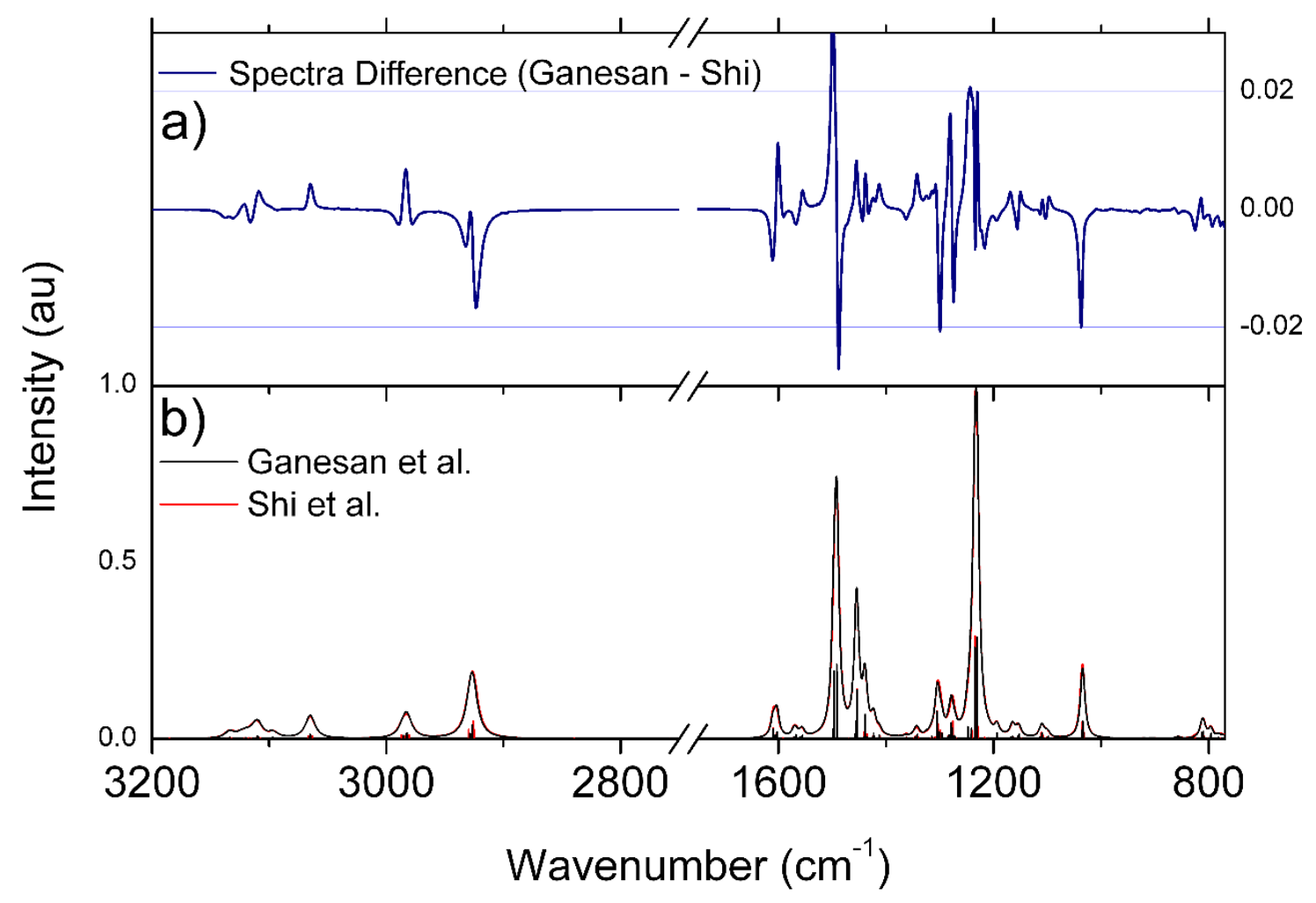

Figure S9. FTIR traces for the theoretical structures calculated from the crystal structures reported by Ganesan et al and Shi et al. a) Spectra difference, b) Histogram and Lorentzian shape curves with $5 \mathrm{~cm}^{-1}$ of FWHW. 
Table S1. Calculated and assigned vibration modes of spiro-MeOTAD using DFT methods.

\begin{tabular}{llll}
\hline Theoretical frequency $\left(\mathrm{cm}^{-1}\right)$ & Vibrational Band Assignment & Bond & Vibration \\
\hline 3133 & Peripheral rings & C-H & Sym stretching \\
3121 & Core rings & C-H & Sym stretching \\
3110 & Peripheral rings & C-H & Asym stretching \\
3097 & Core rings & C-H & Asym stretching \\
3065 & methoxide & C-H & Degenerated stretching \\
2983 & methoxide & C-H & Asym stretching \\
2927 & methoxide & C-H & Sym stretching \\
1609 & Peripheral rings & C-H & bend \\
1602 & peripheral and core rings & C-C & ring strentch \\
1567 & Peripheral rings & C-C & ring deform \\
1555 & Core rings & C-C & ring deform \\
$1496-1492$ & peripheralrings & C-H & rocking \\
1455 & methoxide & C-H & deform \\
1454 & Core rings & C-H & rocking \\
1439 & methoxide & C-H & deform \\
1438 & Core rings & C-H & deform \\
1424 & methoxide & C-H & deform \\
1305 & peripheral and core rings & C-H & rocking \\
1278 & Peripheral rings & C-H & rocking \\
1235 & peripheral and core rings & C-H & rocking \\
1231 & methoxide & C-O & strength \\
1038 & methoxide & C-O & strength \\
810 & Peripheral rings & C-H & wagging \\
795 & Core rings & & \\
\hline & & &
\end{tabular}


Table S2. Four point probe sheet resistance for the four type thin films before and after annealing at $160{ }^{\circ} \mathrm{C}$ to remove crystallization $\mathrm{CH}_{3} \mathrm{Cl}$ molecules. ${ }^{a}$

\begin{tabular}{lll}
\hline Thin film sample & Sheet Resistance $(\Omega / s q)$ & Sheet Resistance (annealed sample) $(\Omega / \mathbf{s q})$ \\
\hline spiro-0 & $-^{-}$ & $-^{-}$ \\
spiro-Li-TBP & $525.5 \pm 0.7$ & $515 \pm 8$ \\
spiro-Li & $440 \pm 40$ & $487 \pm 21$ \\
spiro-TBP & - $^{-}$ & $-^{-b}$ \\
\hline \hline
\end{tabular}

a: arithmetic mean values obtained from 12 measurements and rounding the sample thickness standard deviation to the first significant digit, b: not measurable. 